\title{
The impact of foliar application of salicylic acid on carrot plants (Daucus carota L.)
} under drought stress conditions

\author{
${ }^{1}$ El-Tohamy W.A., H.M. El-Abagy ${ }^{1}$, M.A. Badr² and S.D. Abou-Hussein ${ }^{1}$ \\ ${ }^{1}$ Vegetable Research Department, National Research Centre, 33 El Buhouth St., 12622 Dokki, Giza, \\ Egypt. \\ ${ }^{2}$ Plant Nutrition Department, National Research Centre, 33 El Buhouth St.,12622 Dokki, Giza, Egypt.
}

Received: 30 Oct. 2019 / Accepted 10 Jan. 2020 / Publication date: 30 Jan. 2020

\begin{abstract}
As drought stress can cause a serious effects on growth and productivity of many plants, finding a simple and effective method to minimize the negative effects of drought could be of great advantage in such conditions. The response of carrot plants to foliar application of salicylic acid (SA) under drought stress was tested in this study. Different levels of SA $(0.2,0.4,0.6,1$ and $1.5 \mathrm{~g} / \mathrm{L})$ were applied to carrot plants subjected to drought stress while control plants were not treated by SA. Several measurements were recorded to evaluate the effects of SA on carrot plants under such conditions. The results revealed that the application of SA resulted in reducing the negative effects of drought stress on carrot plants as indicated by higher growth, productivity and quality parameters. A significant improvement of carrot growth, productivity and quality of roots was detected in response to SA application, indicating a better recovery from drought stress when carrot plants were previously treated by SA. The specific effects of SA on carrot growth parameters, yield and quality of roots are discussed.
\end{abstract}

Keywords: carrot (Daucus carota L.), salicylic acid, drought stress

\section{Introduction}

Water becomes a limiting factor for crop production especially in arid and semi-arid regions. Drought results in various injuries to plants including carrot plants. However, Salicylic acid may provide a practical solution for plant protection against drought in such arid regions. There is evidence that this approach may be a potential solution. Anosheh et al. (2012) cleared that the importance of SA and cycocel on reducing drought injury is due to better regulating of stomatal opening, maintenance of higher chlorophyll content of leaves and improvement of water-use efficiency. Also, Lakzayi et al. (2014) stated that SA has an important role in signaling in plants resulting in reducing drought injury of plants. For carrot plants, Ina et al. (2007) indicated that SA had a positive effect on root dry weight of carrot and high carotenoids and anthocyanin content. They also found that SA had an important role in plant response to salinity and osmotic stress as well as its role in regulating the accumulation of proline content in leaves. Moreover, El Tayeb et al. (2010) indicated that SA has an important role in plant signaling during stress and it could have an effective role on improving drought tolerance of wheat plants.

Although the effects of SA on other crops has been studied extensively, little research work was done on the effects of SA on carrot plants. The present study was designed to evaluate the effects of SA on growth, productivity and quality of carrot plants under drought stress conditions.

\section{Materials and Methods}

Seeds of carrot (Daucus carota L.), cv. 'Chantenay' were sown in the winter season of 2016 and 2017 at the National Research Center. The seeds were sown in 5 liter pots (filled with peat moss). Carrot plants (at 3rd leaf stage) were sprayed by salicylic acid $(0.2,0.4,0.6,1$ and $1.5 \mathrm{~g} / \mathrm{L}$ ). One day after treatments, plants were subjected to drought stress by withholding water for 5 days. Control plants were not sprayed by SA but were subjected to drought stress. Tensiometers (Irrometer Company, Riverside, Calif.) were used for soil water potential monitoring. After drought stress period, all plants were re-watered and kept at field capacity to follow post stress observations on 
vegetative growth parameters and yield as well as some quality parameters of roots. All plants were fertilized by using a nutrient solution (15-5-25, NPK) during the entire experiment.

The following parameters were recorded:

Plant height, number of leaves, root length, root diameter and fresh weight of roots and shoots were measured at the end of the experiments.

Total soluble solids (T.S.S.) of roots was also measured by a portable refractometer at the end of the experiments.

Experimental design and statistical analysis: The experiment was designed as a complete randomized block design with 4 replicates. Analysis of variance was calculated according to Snedecor and Cochran (1967). Least significant difference (L.S.D.) at 5\% was used to compare between the means.

\section{Results and Discussion}

Drought stress seriously affects growth, productivity and quality of plants. According to our results, SA improved growth, development and even quality of carrot subjected to drought stress.

SA levels resulted in higher values of plant height and number of leaves compared to control plants (Fig. 1 and 2). The effects were more pronounced at higher levels with maximum effects at 0.6 $\mathrm{g} / \mathrm{L}$ of SA. The higher levels more than $0.6 \mathrm{~g} / \mathrm{L}$ gave lower effects. Similar effects were observed concerning other characters including fresh weight of shoots (Fig. 3) and fresh weight of roots (Fig.4). The results also revealed that carrot plants treated by SA had better recovery from drought. This may be due to the fact that SA is important for plant signaling during periods of stresses including drought (El-Tayeb et al., 2010).

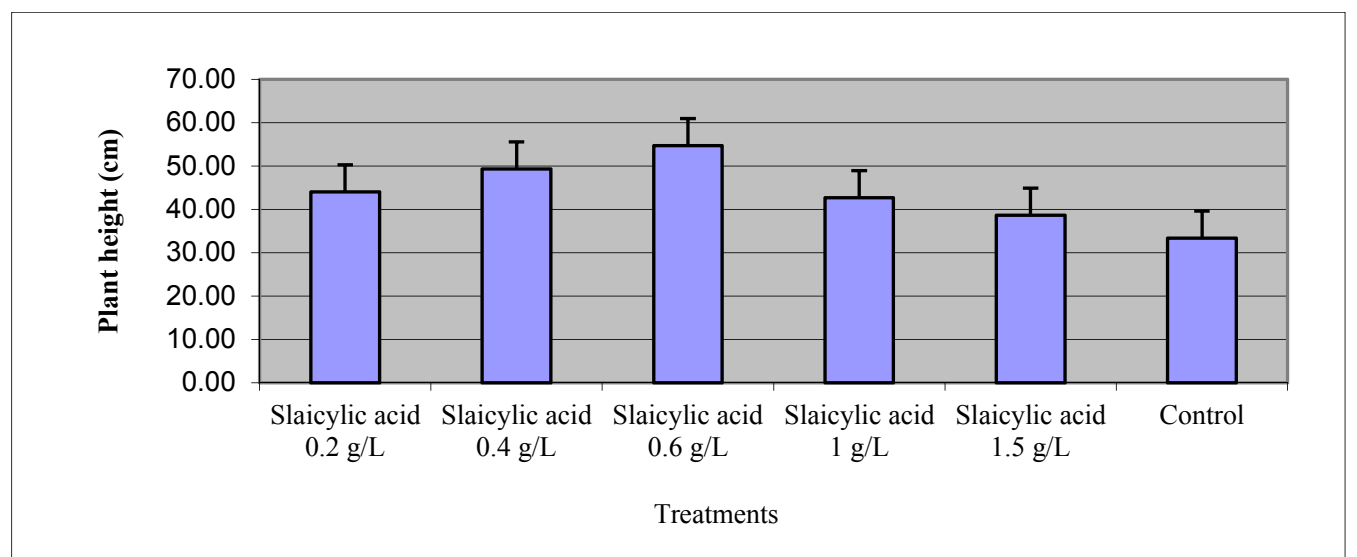

Fig. 1: The effect of salicylic acid on plant height of carrot plants. (Vertical bars present LSD value at $5 \%$.).

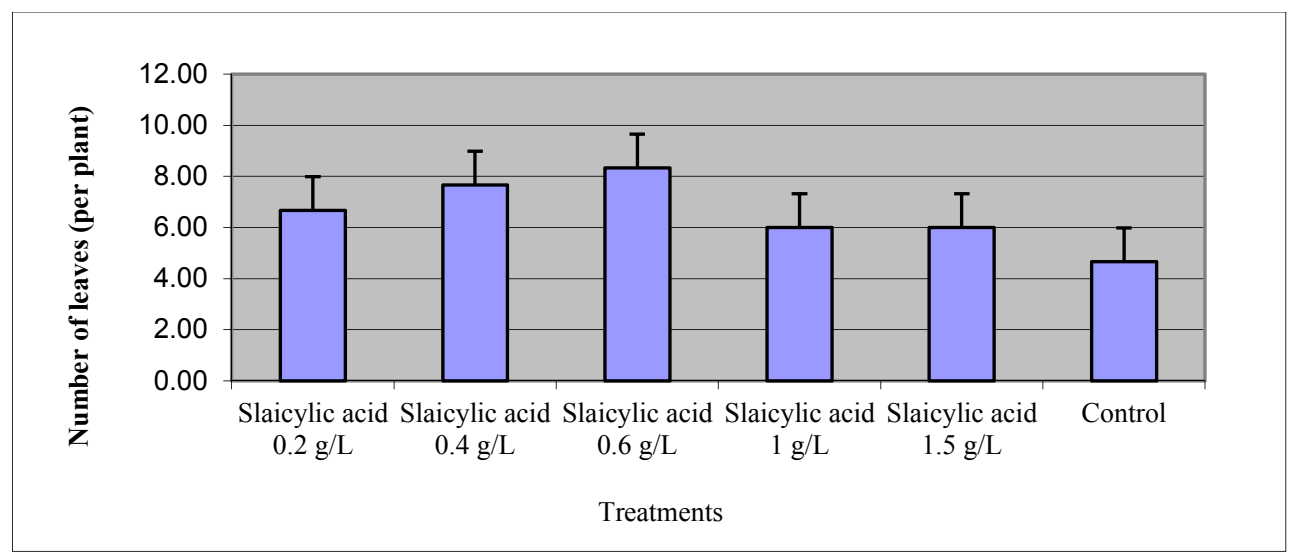

Fig. 2: The effect of salicylic acid on number of leaves of carrot plants. (Vertical bars present LSD value at $5 \%$.). 


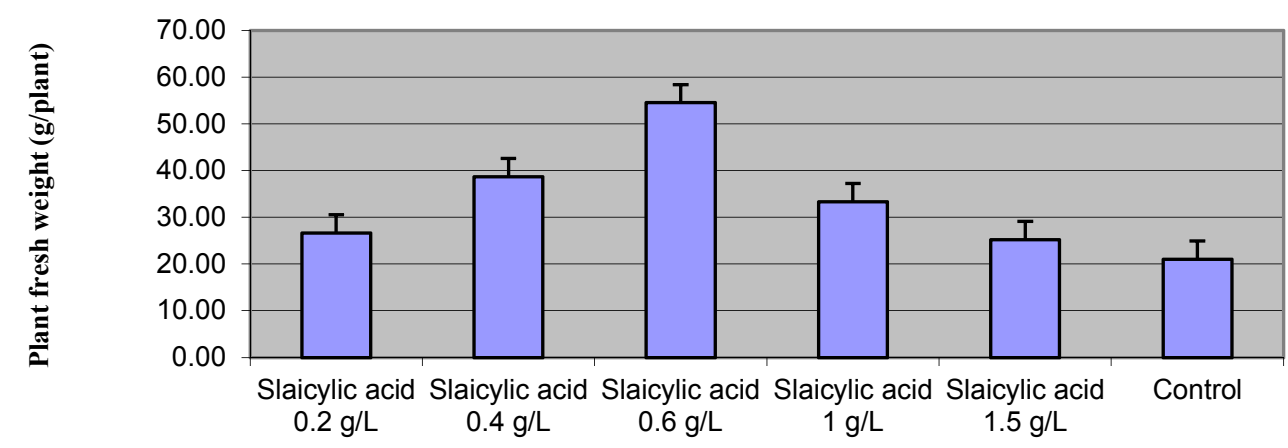

Treatments

Fig. 3: The effect of salicylic acid on fresh weight of shoots. (Vertical bars present LSD value at $5 \%$.).

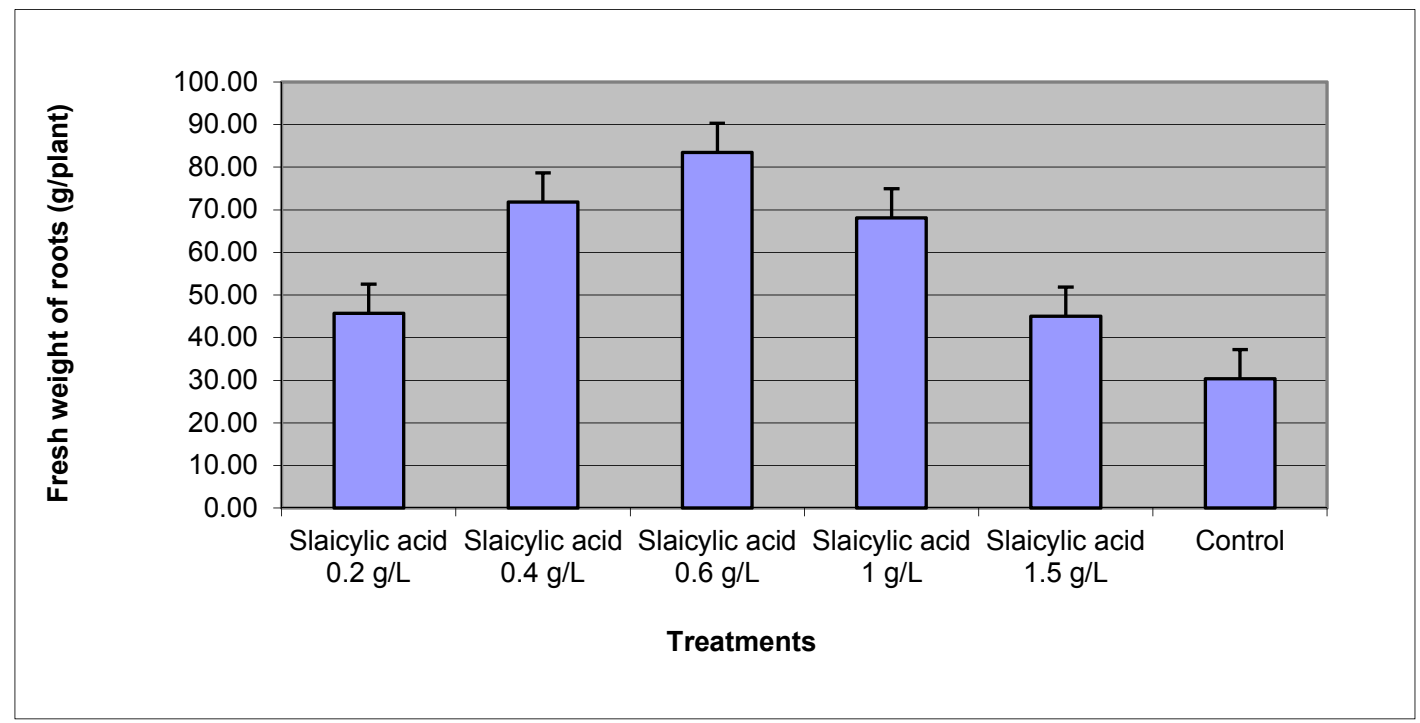

Fig. 4: The effect of salicylic acid on fresh weight of roots. (Vertical bars present LSD value at 5\%.).

According to Ina et al. (2007), SA increased the dry weight of carrot plants and had a positive impact on plant tolerance to osmotic stress. Also, Sorensen et al. (1997) found that drought stress resulted in reducing carrot yield and dry weight of roots when applied at early stages of growth and indicated that severe drought may result in storage loss of roots due to diseases (to make the roots more susceptible to diseases during storage). It was mentioned by Bayat and Sepehr (2012) that foliar application of SA improved drought tolerance of maize plants as growth and productivity were high under such conditions. Moreover, it is worth mentioning that even quality of roots was enhanced by SA application as indicated by high root length (Fig. 5), root diameter (Fig. 6) and total soluble solids of roots (Fig. 7).

Hussain et al. (2008) mentioned that water stress may result in high reduction of yield sunflower plants and that SA application had positive effects on reducing drought injury and improving yield and quality under drought stress conditions. Moreover, the findings of Rao et al. (2012) indicated the efficiency of SA on maintaining high level of RWC of leaves of maize plants subjected to drought stress. On the other hand, Anosheh et al. (2012) cleared that the enhancement of drought tolerance of wheat plants in response to SA application may be due to its effects on maintaining higher chlorophyll content of leaves as well as its effects on improving water-use efficiency.

The results indicated the possibility and the efficiency of using SA as an applicable and safe treatment to enhance carrot growth, productivity and quality under drought stress conditions. 


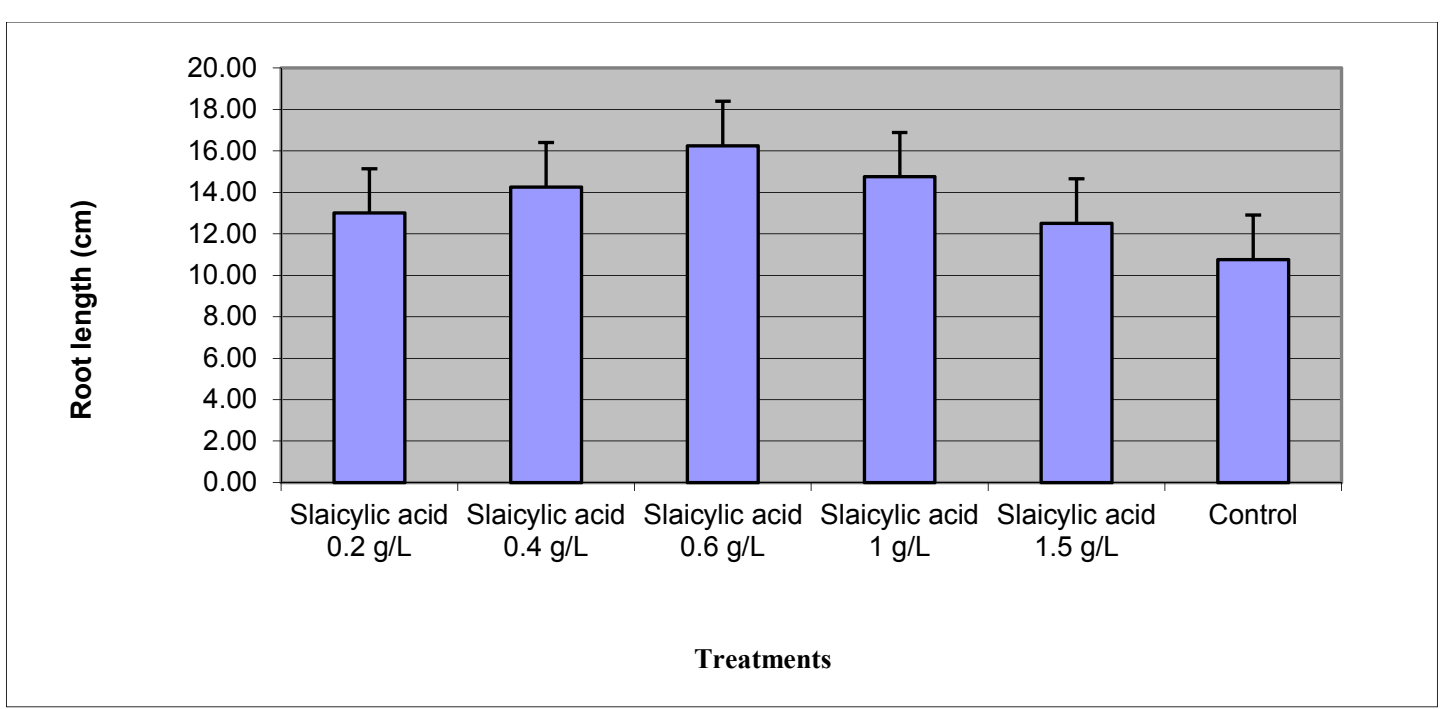

Fig. 5: The effect of salicylic acid on root length. (Vertical bars present LSD value at 5\%.).

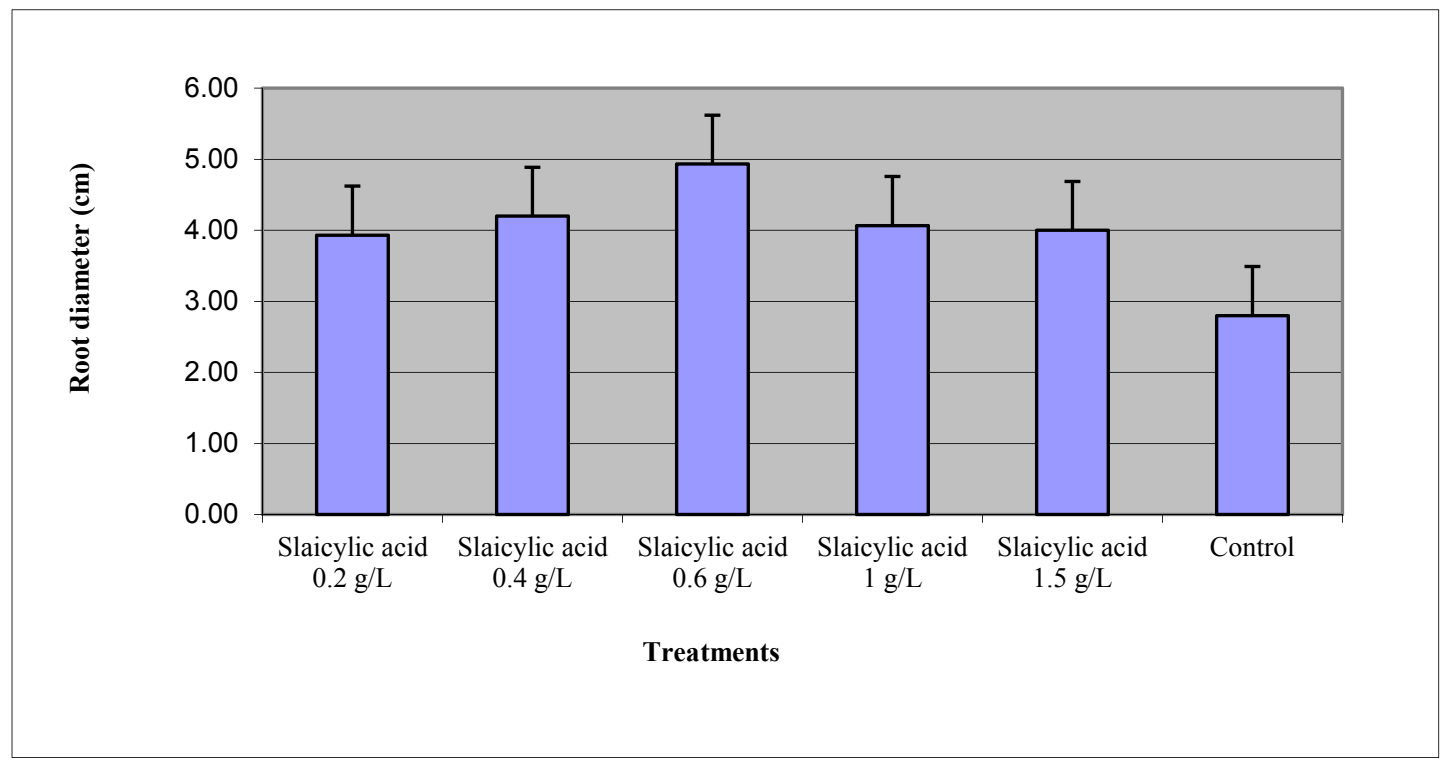

Fig. 6: The effect of salicylic acid on root diameter. (Vertical bars present LSD value at 5\%.).

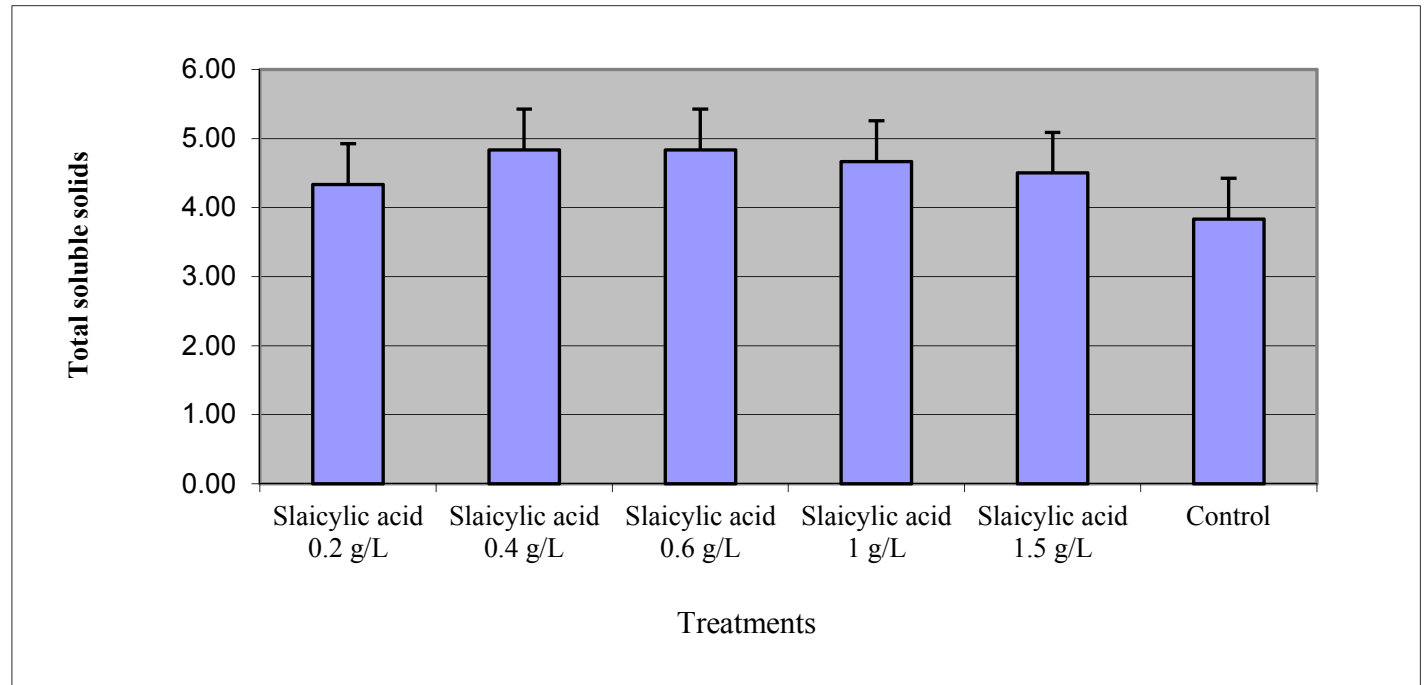

Fig. 7: The effect of salicylic acid on total soluble solids of roots. (Vertical bars present LSD value at 5\%.). 


\section{References}

Anosheh, H.P., Y. Emam, M. Ashraf, and M. R. Foolad, 2012. Exogenous Application of Salicylic Acid and Chlormequat Chloride Alleviates Negative Effects of Drought Stress in Wheat. Advanced Studies in Biology. 4(11), 501-520.

Bayat, S., and A. Sepehr, 2012. Paclobutrazol and Salicylic Acid Application Ameliorates the Negative Effect of Water Stress on Growth and Yield of Maize Plants. Journal of Research in Agricultural Science. 8(2):127- 139.

El Tayeb, M.A., and N. L. Ahmed, 2010. Response of Wheat Cultivars to Drought and Salicylic Acid. American-Eurasian Journal of Agronomy. 3 (1): 01-07.

Hussain, M., Malik, M. A., Farooq, M., Ashraf, M. Y., and M. A. Cheema, 2008. Improving Drought Tolerance by Exogenous Application of Glycinebetaine and Salicylic Acid in Sunflower. Journal of Agronomy and Crop Science.194(3):193-199.

Ina, A., A. Gunes, and M. Alpaslan, 2007. Impact of exogenous salicylic acid on the growth, antioxidant activity and physiology of carrot plants subjected to combined salinity and boron toxicity. Scientia Horticulturae 113:120-128.

Lakzayi, M., E. Sabbagh, K. Rigi, and A. Keshtehgar, 2014. Effect of salicylic acid on activities of antioxidant enzymes, flowering and fruit yield and the role on reduce of drought stress. Intl $\mathbf{J}$ Farm \&Alli Sci. 3 (9): 980-987.

Rao, S. R., A. Qayyum,A. Razzaq, M. Ahmad,I. Mahmood, ,and A. Sher, ,2012: Role of foliar application of salicylic acid and L-Tryptophan in drought tolerance of maize. The Journal of Animal \& Plant Sciences. 22(3):768-772.

Snedecor, G.W., and W.G. Cochran, 1967: Statistical methods, $6^{\text {th }}$ ed. Iowa State Univ. Press, Ames, Iowa, USA.

Sorensen, J.N., U. Jorgensen, and B.F. Kuhn, 1997: Drought effects on the marketable and nutritional quality of carrots. Journal of the science of food and agriculture. 74(3):379-391. 